\title{
PROYECTO DE RESOLUCIÓN TÉCNICA: NORMAS SOBRE LA ACTUACIÓN DEL CONTADOR PÚBLICO EN LA JUSTICIA COMO PERITO
}

$20^{\circ}$ JORNADA NACIONAL DE JÓVENES PROFESIONALES EN CIENCIAS ECONÓMICAS. "EMPRENDIENDO DESAFÍOS PARA UN MUNDO EN CONSTANTE CAMBIO" POSADAS, MISIONES, ARGENTINA 17, 18 Y 19 DE AGOSTO DE 2017

GASTÓN CABRAL

Resistencia, Chaco

ARGENTINA 


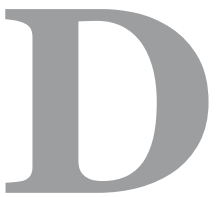

esde el punto de vista legal del ejercicio profesional, afirmamos que el perito contador debe actuar sobre la base de normas de auditoría, ya que, como lo estipulan las leyes de ejercicio profesional, en la emisión de dictámenes se deben aplicar las normas de auditoría aprobadas por los organismos profesionales cuando ello sea pertinente.

Sin embargo, en muchos casos, quienes se desempeñan como peritos, no hacen referencia a esta situación, ni aplican criterios y procedimientos uniformes respecto de la tarea profesional que realizan y/o de su alcance, situación que podría incrementar injustificadamente su responsabilidad, así como también afectar la confiabilidad de su actuación.

Esto implica la necesidad de establecer preceptos profesionales que rigen la función del contador como perito y que haga posible su juzgamiento a la luz de parámetros que hacen a la condición de profesionalidad, como son la aplicación de normas técnicas, amplitud e independencia de criterio y actuación ética.

\section{JUSTIFICACIÓN DEL TEMA}

Con el avance del conocimiento y en plena sociedad de la información, la prueba pericial contable ha tomado gran importancia como medio técnico-científico para auxiliar a la justicia en la búsqueda de la verdad y contribuir a la difícil tarea de los Jueces de arribar a la más adecuada conclusión en cada proceso. Más aún en el contexto vigente, en el cual "una de las características más distintivas y salientes de la actividad empresarial y comercial actual es la creciente complejidad en las operaciones de los negocios y en el funcionamiento operativo de las actividades relacionadas con los movimientos de financieros y económicos entre los grupos empresarios no solo en el ámbito local como así también en el orden nacional e internacional fundamentalmente debido al fenómeno de la globalización y transnacional de sus operaciones debido a los avances en materia de las comunicaciones y la informativa”. ${ }^{1}$

La tarea de los peritos contadores sirven a los magistrados para fundar sentencias con responsabilidad social para con el comercio y la sociedad en su conjunto. Especialmente, en los procesos en los cuales se ven afectadas unidades económicas que tienen un impacto significativo en las comunidades en las que están insertas. Porque las sentencias, en una sociedad judicializada como la que tenemos, son una variable económica pocas veces estudiada que tiene implicancias para las economías regionales.

\footnotetext{
${ }^{1}$ DELL'ELCE, Q. “Algunos aspectos importantes a tener en consideración previo a ordenar un estudio pericial contable”; trabajo presentado ante las XX Jornadas de Actuación Judicial organizadas por el CGCECF y en las X Jornadas de Investigación y Reflexión en Cátedra, FCE - UBA. 2008. Pág 12.-
} 
El gran valor probatorio de las pruebas periciales hacen que sean ofrecidas voluntariamente por las partes, pudiendo estas decidir si participaran de las mismas. Es por ello que "el ofrecimiento de este tipo de pruebas toma cada vez mayor importancia para acreditar los extremos pecuniarios incoados en las demandas". ${ }^{2}$

Asimismo, a la fecha no existen normas específicas relativas a la actuación del contador como perito, razón por la cual, esperamos que este trabajo sea un comienzo para dar respuesta a esa situación.

\section{OBJETIVOS}

(...) En cuanto a la investigación, buscamos confirmar si los colegas que participan en las comisiones asesoras de actuación judicial de los Consejos Profesionales de Ciencias Económicas de Chaco y Corrientes tienen interés en la emisión de un marco normativo respecto de la actuación de los contadores públicos como peritos en la justicia. Como así también, validar los ejes que debería tratar la norma, considerando cuestiones técnicas de la profesión, salvando las cuestiones procesales que se encuentran reservadas para cada una de las jurisdicciones, en los respectivos códigos de forma.

Ahora bien, con respecto a la resolución técnica en sí, creemos que la emisión de una norma como la que se proyecta, permitiría un conocimiento más acabado de jueces y organismos de control y profesionales que tuvieran que interpretar la labor del perito y valorar su actuación, constituyéndose en parámetros objetivos. Además, posibilitaría sintetizar en un único documento los distintos avances y trabajos que existen actualmente al respecto.

Es por ello que, "a los efectos de elevar el nivel del desempeño profesional del perito judicial actuante, es conveniente establecer pautas de actuación más claras y amplias que regulen y faciliten el cumplimiento de sus funciones específicas". ${ }^{3}$

A nivel formativo, la norma técnica se incluiría en los planes de estudio de las facultades de ciencias económicas, otorgándole una entidad a esta incumbencia que reforzaría la formación en este sentido.

Una norma de este estilo, contribuiría a crear el marco referencial proporcionando a la matrícula herramientas útiles para el desarrollo de la labor que debe encarar en los casos de desempeñarse como perito en la justicia.

\footnotetext{
2CABRAL, G. Ponencia: "Reflexiones de jóvenes peritos". XVIII Jornada Nacional de Jóvenes Profesionales de Ciencias Económicas FACPCE. 2013. Pág. 6.-

${ }^{3}$ DELL’ELCE. Q. “Informe № 3 C.E.C.yT.- Área Judicial y Societaria - Contenido y Estructura de los dictámenes periciales contables. Disposiciones normativas relacionadas. 2002. Pág. 19.-
} 


\section{SUPUESTOS INICIALES}

Al finalizar este trabajo, trataremos de validar estos supuestos:

a) Que no existe una norma específica para la actuación del contador en la justicia como perito y que la profesión necesita un marco referencial que sustente sus actuaciones.

b) Que el contenido de dicha resolución, deberá abarcar los siguientes ejes: Antecedentes; Alcance y responsabilidad profesional; Condición básica para el ejercicio como perito; Normas para la actuación del perito; Documentación de la tarea profesional; Normas sobre informes; Guías de tareas sobre puntos periciales recurrentes y Modelos de informes y actas.

\section{METODOLOGÍA DE INVESTIGACIÓN}

El objeto de investigación estará constituido por la actuación pericial de los contadores públicos en la justicia y será estudiado a partir de los datos recolectados a través de charlasdebates en comisión con los matriculados de los C.P.C.E. de Chaco y Corrientes, aplicando técnica de sondeo. Los aportes de los colegas fueron plasmados en encuestas y anotaciones por cada encuentro.

Además, realizamos una búsqueda de antecedentes entre los Informes técnicos del C.E.C.yT., Memorandos vigentes de Secretaria Técnica de la F.A.C.P.C.E., Trabajos de investigación aprobados en los Congresos 2012, 2014 y 2016 de la F.A.C.P.C.E., dentro del área de actuación en la justicia, y análisis de trabajos varios sobre la temática localizados a través de buscadores por Internet, a los fines de recopilar y contrastar el material teórico existente sobre esta incumbencia con la práctica profesional de los colegas en la región.

Las opiniones vertidas en cada encuentro constituyen datos empíricos de fuente primaria sobre los cuales se apoya la conclusión del presente (...).

\section{DESARROLLO}

Como mencionamos, los resultados que consignamos surgen de la búsqueda, recopilación y análisis bibliográfico sobre el tema y de los encuentros que realizamos con los colegas el 23 de mayo de 2017 en el C.P.C.E. de Corrientes y el 31 de mayo de 2017 en el C.P.C.E. de Chaco.

En los mismos, participaron un total de 10 (diez) colegas que ejercen en la justicia. Sin bien, a primera vista parecería una cantidad poco representativa, en nuestra zona, la participación de dicha cantidad de matriculados es aceptable. 
Con ellos, debatimos el contenido del presente trabajo y cuyas conclusiones exponemos a continuación.

\section{ANTECEDENTES}

De la búsqueda de antecedentes que realizamos entre documentos del C.E.C.yT., Secretaría Técnica de la F.A.C.P.C.E., los últimos tres Congresos Nacionales y análisis bibliográfico destacamos los siguientes como antecedentes que podrían servir de sustento para una futura resolución técnica que contenga normas de actuación para peritos:

- La Resolución Técnica $\mathrm{N}^{\circ} 37$ F.A.C.P.C.E.

- Resolución Técnica $\mathrm{N}^{0} 15$ (t.a. R. T. 45) F.A.C.P.C.E.

- Memorando de Secretaria Técnica de la F.A.C.P.C.E. No C-3: Validez técnica y legal de Estados Contables que no surgen de libros rubricados. Bs. As. 07/11/1988 (actualizado a agosto 2014)

- Memorando de Secretaría Técnica de la F.A.C.P.C.E. No A-38: Obligatoriedad de informar en el informe del auditor si los estados contables surgen de registros contables llevados en sus aspectos formales de legal forma. Bs. As. 13/10/1998 (actualizado a agosto 2014)

- Informe $\mathrm{N}^{0} 3$ C.E.C.yT.- Área Judicial y Societaria - Contenido y Estructura de los dictámenes periciales contables. Disposiciones normativas relacionadas. 2002 Dr. Quintino Pierino Dell'Elce.

- Trabajo: Dictamen profesional sobre los registros contables. Algunos aspectos importantes que se sugieren tener en especial consideración al momento de ordenar la prueba pericial contable de parte de los magistrados judiciales. XXXII Simposio Nacional de Profesores de Práctica Profesional. Universidad Nacional de Santiago del Estero. Termas de Río Hondo, 23 y 24 de septiembre de 2010. Dr. Quintino Pierino Dell'Elce

- Ponencia: Reflexiones de jóvenes peritos. XVIII Jornada Nacional de Jóvenes Profesionales de ciencias económicas: "Buscando respuestas a los nuevos desafíos de nuestra realidad” Corrientes, 26, 27 y 28 de septiembre de 2013. Dres. Gastón Cabral y Anahí Agustini.

Durante los encuentros, la totalidad de los colegas nos manifestaron que no tenían conocimiento de antecedentes técnicos como los mencionados precedentemente y consideraron que los mismos tienen relevancia para el fin propuesto. 


\section{ALCANCE Y RESPONSABILIDAD PROFESIONAL}

En este apartado, pretendemos aclarar y delimitar el alcance que tendría la resolución, proponiendo que comprenda la actuación del contador público como perito en toda su extensión, llámese perito contador, asesor, consultor técnico, liquidador, partidor, auditor, entre otros.

Abarcando tanto a los peritos que actúen de forma única o como integrante de un equipo, designado de oficio o también propuestos de parte, resaltando que actuar de parte no significa que el perito deba apartarse de las normas éticas por el hecho de haber sido propuesto de forma unilateral por una de las partes.

Antes de avanzar, queremos distinguir que "gran parte de las consideraciones que se comentan son también extensivas y aplicables...a los licenciados en administración y...economía”; quienes podrán encontrar aquí un puntapié para normar su actuación como peritos.

Ahora bien, como regla general, el perito debe cumplir con las obligaciones profesionales a partir del momento en que acepta expresamente la designación judicial y hasta la finalización de la misma o efectúe una renuncia fundada, lo que ocurra primero.

Algunos autores sostienen que la designación es una carga pública y como tal, no es fácilmente renunciable, pero lo cierto es que siguiendo el artículo $6^{\circ}$ del código de ética unificado aprobado por resolución 204/2000 de la F.A.C.P.C.E. sostenemos que "los profesionales deben evitar la acumulación o aceptación de cargos, funciones, tareas o trabajos profesionales que excedan sus posibilidades de cumplimiento (...)”.

Con este criterio estamos en condiciones de afirmar que la renuncia hecha bajo este parámetro, no constituye una irresponsabilidad del profesional, sino todo lo contrario, sería un acto responsable conocer las limitaciones que cada uno tiene.

En los encuentros, los colegas manifestaron que los ámbitos de actuación del contador son tan variados que no todos los podemos manejar en su totalidad. Hay quienes se abocan a la parte laboral y previsional, otros societaria, etc. Y en ciertas circunstancias reconocer las limitaciones que uno podría tener en cuanto el trabajo a realizar, es un acto prudente que no debe ser castigado con alguna sanción (...)

Para finalizar, entendemos que la responsabilidad del perito en lo relativo a su labor, no se extiende en los casos de proporcionarse documentación apócrifa, excepto que la documentación adulterada, que fuera objeto del encargo del perito, presentará rasgos flagrantes e inequívocos de invalidez, fácil e indudablemente perceptibles.

Retomando las conclusiones de los debates, decimos que el 30\% de los colegas manifestaron estar plenamente conformes con lo expuesto. Mientras que un 10\% prefirió no contestar; y el $60 \%$ restante, indicó su conformidad pero consideraron necesario continuar con la investigación.

${ }^{4}$ DELL’ELCE. Q. “Informe № 3 C.E.C.yT.- Área Judicial y Societaria - Contenido y Estructura de los dictámenes periciales contables. Disposiciones normativas relacionadas. 2002. Pág. 5- 


\section{INDELEGABILIDAD DEL CARGO}

Por aplicación del artículo 10 del código de ética unificado, decimos que el cargo de perito es personal e indelegable.

No obstante, algunas tareas procesales, como ser: notificaciones, diligenciamientos de escritos, retiro de documental, procuraduría del expediente y trámites ante demás organismo de control, pueden requerir la delegación de tareas en colaboradores con la debida supervisión y control, debiendo el perito designado asumir finalmente la responsabilidad total por las actuaciones judiciales.

Dicha delegación deberá ser debidamente informada a V.S. con la suficiente antelación para no dar lugar a objeciones de ningún tipo.

De forma similar, en este caso, el $40 \%$ se mostró conforme. Nuevamente un 10\% prefirió no contestar; y el 50\% restante, indicó su conformidad con la salvedad de profundizar la investigación.

\section{CONDICIÓN BÁSICA PARA EL EJERCICIO COMO PERITO}

\section{Independencia}

La independencia es la condición básica que se deriva de la aplicación de las normas de auditoría vigentes. Es por ello que el perito debe ser independiente.

Pero ahora nos preguntamos ¿̇Independiente de quien? Del Juez, los abogados patrocinantes, las partes, los terceros citados en garantía y del ente cuya documentación sea objeto del proceso?

En los encuentros concluimos que a fin de cumplir con las normas de auditoría vigentes, el perito debe ser independiente de las partes y del ente cuya documentación sea objeto de la prueba pericial.

Por otra parte, quedará librado a cada jurisdicción la interpretación de la jurisprudencia y normas legales vigentes sobre la excusación para la actuación respecto de abogados, apoderados y magistrados, aplicándose en cada caso, la disposición más restrictiva (...).

\section{Alcance de las incompatibilidades}

Siguiendo el criterio de compatibilidad que establecen las R.T. 37 y 15, en cuanto a la teneduría de libros y auditoría externa, adherimos y decimos que las funciones de perito son compatibles con otros servicios profesionales realizados por el contador público, en la medida que estos cumplan con las condiciones de independencia expresados en el apartado anterior. 
El hecho de prestar servicios continuos y retribuidos por medio de honorarios no genera relación de dependencia con los comitentes.

Por ello, afirmamos que las funciones del perito son compatibles con la teneduría de libros, la auditoría externa y asesoría fiscal.

Podrá variar la naturaleza, alcance y oportunidad de los procedimientos a aplicar en el cumplimiento de uno y otro servicio profesional pero, básicamente, los objetivos son similares y nada impide que dichas prestaciones puedan recaer en un mismo contador público.

Es por ello que, desde el punto de vista de la profesión de contador público, se ha venido interpretando y sosteniendo que, en el cumplimiento de sus tareas, el perito actúa sobre la base de las normas de auditoría de los organismos profesionales en todo aquello que hace a controles sobre la existencia de activos, sobre la documentación respaldatoria de operaciones, sobre la revisión de registros contables y, fundamentalmente, a la preparación de su informe sobre información contable de los entes.

A ello debe agregarse que la sola circunstancia de que el perito pueda ser, además, auditor externo o asesor fiscal, en nada perjudicará su independencia.

En todo caso, no es la mera y eventual superposición de tareas la que genera el conflicto de intereses o vulnera la independencia profesional.

Ahora bien, de tal acumulación decimos que refuerza la función del perito porque profundiza el conocimiento de la estructura del sistema contable del ente y resulta consecuentemente valiosa para el proceso judicial (...).

Quizás en este punto, el lector se encuentre desorientado, pero este apartado resulta clave para avanzar en las competencias del profesional para expedirse sobre la forma legal de los libros y "la representatividad económica patrimonial que deben reflejar dichos registros" 5 ; pregunta recurrente en los pliegos periciales. Siendo dicha representatividad una cuestión de fondo que es resuelta por los contadores al momento de auditar los balances del ente y que luego podría recaer nuevamente en un contador que haya participado en la auditoría externa de períodos pasados o se encuentre prestado algún servicio profesional en el presente.

$\mathrm{Al}$ momento de responder sobre la forma legal de los libros "en general no es común ni frecuente advertir que se consigne de parte del profesional interviniente alguna opinión o consideración específica sobre los aspectos sustanciales en que aparecen ser llevados los registros contables examinados durante el cumplimiento de su labor profesional ni mucho menos acerca de la validez, veracidad y confianza que pudieran ellos merecer como efecto de la información consignada que surja de las propias anotaciones y transcripciones asentadas en ellos". ${ }^{6}$

\footnotetext{
${ }_{5}^{5}$ CABRAL, G. Ponencia: "Reflexiones de jóvenes peritos". XVIII Jornada Nacional de Jóvenes Profesionales de Ciencias Económicas FACPCE. 2013. Pág. 6.-

${ }^{6}$ DELL'ELCE, Q. “Algunos aspectos importantes a tener en consideración previo a ordenar un estudio pericial contable”; trabajo presentado ante las XX Jornadas de Actuación Judicial organizadas por el CGCECF y en las X Jornadas de Investigación y Reflexión en Cátedra, FCE - UBA. 2008. Pág. 9.-
} 
Nos ceñimos a las formas y no advertimos al Juez que "la validez técnica contable no se altera si los registros contables están rubricados o no. Los asientos son idénticos en ambos casos y los medios de registración también...por tal razón, en determinadas situaciones un estado contable que surja únicamente de documentos respaldatorios podría prevalecer sobre un estado contable que surja de registros rubricados si estos últimos no están respaldados por documentación".7

Con esto no queremos minimizar las formalidades, ya que estas hacen al valor probatorio de la documentación, pero dependiendo el caso, algunas omisiones formales, en determinados casos, podrían llegar a subsanarse con procedimientos alternativos.

Las cuestiones formales de los registros (rúbrica, encuadernación, etc.) "tienen el propósito de individualizar dichos balances y garantizar el registro cronológico de los mismos,

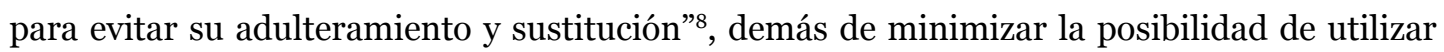
balances distintos para un mismo periodo, los cuales son falseados según el usuario de la información al que estén destinados, conocidos como balances mellizos (...).

A lo dicho, debemos agregar que en los debates, las condición básica para el el ejercicio, demostró un acuerdo del 70\%, mientras que el 30\% restante expresó su disconformidad al respecto.

\section{Normas para la actuación del perito}

La actuación del perito la podemos englobar en dos grandes categorías. Por un lado, la compulsa y por el otro, la pericia propiamente dicha.

Definimos a la compulsa judicial como el procedimiento que consiste en cotejar una copia o constatar algún dato preciso, con documentación original para determinar su exactitud. Dicha documentación debe estar relacionada a la contabilidad, de allí la justificación para que el procedimiento sea realizado por un contador.

En cambio, entendemos que la peritación es una declaración técnico-científica, mediante la cual, el profesional expone las conclusiones de los hechos sobre los que versa su dictamen, ya sea por percepción, deducción o inducción, luego de aplicar su disciplina, sin efectuar valoraciones jurídicas.

Las leyes arancelarias hacen esta distinción. La cual consideramos oportuna plasmarla porque permitirá a los Jueces valorar adecuadamente la retribución del trabajo profesional al momento de la regulación de honorarios y a los colegas encuadrar debidamente su labor.

El perito, para cumplir su manda judicial debe aplicar los procedimientos establecidos en las normas de auditoría vigentes. Por lo tanto, la naturaleza, alcance y oportunidad de su tarea dependerá de los puntos de pericia a resolver, las circunstancias particulares del caso, el riesgo involucrado y el criterio profesional.

\footnotetext{
${ }^{7}$ F.A.C.P.C.E. Memorando de Secretaria Técnica № C-3: "Validez técnica y legal de Estados Contables que no surgen de libros rubricados. actualizado 2014. Pág. 1

${ }^{8}$ CABRAL, G. Ponencia: "Reflexiones de jóvenes peritos". XVIII Jornada Nacional de Jóvenes Profesionales de Ciencias Económicas FACPCE. 2013. Pág. 7.-
} 
El perito, a través del desarrollo de su tarea, debe obtener elementos de juicio válidos y suficientes que permitan respaldar las aseveraciones formuladas en sus informes.

Este aplicará los procedimientos de auditoría sobre bases selectivas, determinadas según su criterio o apoyándose en el uso de métodos estadísticos y, orientará su trabajo en función de la significatividad que los datos o hechos puedan tener.

En los encuentros, el debate arrojó un 100\% de adhesión a este punto.

\section{Documentación de la tarea profesional}

"En la tarea de campo el perito debe recabar la evidencia necesaria para responder a las preguntas del cuestionario". 9

En consecuencia, el perito debe documentar adecuadamente el trabajo realizado, es decir, hacerlo tangible.

De ahí que la mejor forma de exteriorizar dicha labor es por medio de actas que describan la situación que se presenta. Por este motivo, en el anexo 2 proporcionamos un modelo a tal fin.

Además, resulta conveniente al momento de recibir información contable del ente, que la misma sea debidamente firmada en nuestra presencia, a efectos de acreditar la procedencia de la misma (...).

\section{Contenido de los papeles de trabajo}

Los papeles de trabajo deberían contener mínimamente lo siguiente:

- La descripción de la tarea a realizar.

- La asignación de tareas y responsables, en caso de haber más de un perito.

- Los datos y antecedentes recogidos durante el desarrollo de la tarea, ya se tratare de aquellos que el perito hubiere preparado o de los que hubiere recibido de terceros.

- El lugar y fecha de realización.

- Las conclusiones particulares y generales.

El perito debe realizar su tarea dentro del principio de economía aplicable a toda auditoría. $O$ sea, para que su labor sea económicamente útil debe culminar en un lapso y a un costo razonable.

El trabajo del perito debería realizarse de acuerdo con lo previsto en una resolución técnica específica como venimos sosteniendo, y en lo pertinente, con las normas de auditoría establecidas en la Resolución Técnica $\mathrm{N}^{\circ} 37$ de la F.A.C.P.C.E.

Es preciso señalar que no es una obligación del perito adjuntar los papeles de trabajo al informe, pero que los mismos son un instrumento de defensa ante la necesidad de tener que respaldar su actuación frente a un eventual requerimiento judicial o de organismos de contralor profesional. 
De igual forma, sostenemos que el informe del perito no debe acompañar documentación del ente, copias, testimonios, ni transcribir información que deba aportarse como prueba documental, o tenga que solicitarse por medios de prueba informativa, o que por sus características, deba consultarse en la sede del ente.

(...)

$\mathrm{Al}$ igual que en el apartado anterior, el 100\% de los colegas que asistieron a ambos debates compartieron lo expresado en su totalidad.

\section{Normas sobre informes}

Los informes deberían cumplir con los requisitos o características de la información, establecidos en la Resolución Técnica 16. En especial, se deben evitar los vocablos o expresiones ambiguas o que pudieran inducir a error a los interesados en el dictamen (...).

\section{Contenido del informe del perito}

Los informes escritos deberían contener:

- La identificación del objeto del examen o revisión.

- La indicación de los elementos exhibidos, vistos y analizados.

- El detalle de la tarea realizada, con mención a los principales procedimientos aplicados (...). expresar aquellas procedimientos de auditorías trascendentes y relevantes para el informe.

- La inclusión de párrafos ilustrativos que indiquen de quién es la responsabilidad de los documentos examinados o revisados, el objetivo de una pericia desarrollada en el marco de una auditoría y el alcance de la función del perito.

- La conclusión y/o opinión sobre la información contable objeto de la pericia que ha podido formarse el perito a través de la tarea realizada.

- Los elementos adicionales necesarios para su mejor comprensión (...).

- Los informes deben titularse "Informe del Perito...." ya que este es el término que se emplea en otros trabajos profesionales y que consideramos debemos mantener para ser coherentes con las normas existentes (...).

- Dirigir el informe al Juez.

- Afirmar que los documentos examinados/revisados son responsabilidad del ente.

- Aclarar la responsabilidad que asume el perito respecto de la documentación puesta a consideración.

- Describir sucintamente ciertos aspectos de las normas de auditoría tales como, el marco técnico y ético profesional aplicable, el carácter selectivo de las pruebas, la necesidad de la planificación de la labor, la adecuada evaluación de las estimaciones efectuadas por el ente, y la circunscripción del examen a la información contable significativa. 
- Afirmar que el examen realizado incluye la verificación de la congruencia de las afirmaciones hechas por las partes con la restante información obtenida a través de la pericia contable.

- No deberá aconsejar curso de acción alguno a las partes respecto del proceso.

- Deberá abstenerse de opinar sobre proyecciones y cualquier otra afirmación sobre hechos futuros (...).

Por lo que se refiere a la opinión vertida en los encuentros, subrayamos que el 90\% consideró apropiado el contenido que proponemos para el informe pericial.

\section{CONCLUSIÓN}

Al comienzo del trabajo nos propusimos conocer el interés de la matrícula en dictar una norma técnica, para lo cual realizamos dos charlas, en las cuales debatimos con los colegas sobre esta cuestión. En ambas concluimos que el 100\% de los asistentes tiene un interés profesional en el dictado de una norma técnica.

Si bien, la idea original era proyectar una resolución técnica, solo el 60\% de los asistentes a los encuentros adhirió a esta posibilidad, mientras que el otro $40 \%$ consideró más conveniente el dictado de una recomendación, para que su aplicación no sea obligatoria atendiendo a las distintas realidades que posee esta incumbencia en las distintas provincias.

No obstante, resulte una resolución o una recomendación técnica, la totalidad de los colegas compartieron el contenido propuesto y se mostraron entusiastas al afirmar que la emisión de un documento constituirá un comienzo para afianzar la formación en las facultades.

A modo de conclusión final, estamos conformes con los resultados que arrojó la investigación en cuanto a los ejes y contenidos sobre los cuales debería pasar la discusión profesional.

Asimismo, vemos como algo muy positivo que exista un interés en trabajar sobre normas profesionales que sigan construyendo y jerarquizando nuestra profesión.

\section{REFERENCIAS BIBLIOGRÁFICAS}

Resolución Técnica $\mathrm{N}^{\circ} 37$ - F.A.C.P.C.E.

Resolución Técnica No 15 (t.a. R.T. 45)

Memorando de Secretaria Técnica de la F.A.C.P.C.E. N ${ }^{\circ}$ C-3: Validez técnica y legal de Estados Contables que no surgen de libros rubricados. Bs. As. 07/11/1988 (actualizado a agosto 2014) 
Memorando de Secretaría Técnica de la F.A.C.P.C.E. No A-38: Obligatoriedad de informar en el informe del auditor si los estados contables surgen de registros contables llevados en sus aspectos formales de legal forma. Bs. As. 13/10/1998 (actualizado a agosto 2014).

Informe $\mathrm{N}^{\mathrm{o}} 3$ C.E.C.yT.- Área Judicial y Societaria - Contenido y Estructura de los dictámenes periciales contables. Disposiciones normativas relacionadas. 2002 Dr. Q. P. Dell’Elce.

Trabajo: Dictamen profesional sobre los registros contables. Algunos aspectos importantes que se sugieren tener en especial consideración al momento de ordenar la prueba pericial contable de parte de los magistrados judiciales. XXXII Simposio Nacional de Profesores de Práctica Profesional. Universidad Nacional de Santiago del Estero. Termas de Río Hondo, 23 y 24 de septiembre de 2010. Dr. Q. P. Dell’Elce.

Ponencia: Reflexiones de jóvenes peritos. XVIII Jornada Nacional de Jóvenes Profesionales de ciencias económicas: "Buscando respuestas a los nuevos desafíos de nuestra realidad" Corrientes, 26, 27 y 28 de septiembre de 2013. G. Cabral y A. Agustini.

Resolución 204/2000 - Código de ética unificado F.A.C.P.C.E.

Nedel, O. “Manual pericial: la actuación del contador público en la justicia”. 1991.

\section{NOTA EDITORIAL}

El presente es una reseña del trabajo completo que se encuentra disponible en http:// www.20jornadasjovenes.com/trabajos/ Asimismo, lo expresado en la investigación, no constituye una opinión de la Federación Argentina de Consejos Profesionales de Ciencias Económicas, ni de ningún Consejo adherido. Derechos reservados. FACPCE. 2017.-

\section{CURRICULUM VITAE}

\section{Gastón Maximiliano Cabral}

Contador Público.

UNNE. MP 3021 - CPCE Chaco.

Profesional Independiente. Perito.

gaston.cabral@gmail.com 